\title{
STRUCTURAL OPTIMIZATION USING ARTIFICIAL IMMUNE SYSTEM (AIS)
}

\author{
Sai Sushank Botu ${ }^{1}$, S V Barai $^{2}$ \\ ${ }^{1}$ Department of Civil Engineering, Indian Institute of Technology Kharagpur, Kharagpur, \\ India \\ ${ }^{2}$ Department of Civil Engineering, Indian Institute of Technology Kharagpur, Kharagpur, \\ India (skbarai@,civil.iitkgp.ernet.in)
}

\begin{abstract}
In this paper a new evolutionary computational paradigm - Artificial Immune System (AIS) is explored for the optimal design of truss structures. CLONal selection ALGorithm (CLONALG) which is imitated by the basic principle of adaptive immune response to the virus stimulus is one of the extensively used models of AIS. Since optimal design of truss structures involves multi modal search space and being CLONALG proved very effective in solving multi modal optimization problems, the algorithm is employed for the task of optimization. The effectiveness of the algorithm is demonstrated for 2D/3D truss problems. The results are compared with other conventional mathematical approaches and evolutionary techniques.
\end{abstract}

Keywords: Evolutionary Computation, Artificial Immune System, Structural Optimization, Truss Structures

\section{INTRODUCTION}

Structural optimization is a vital and demanding activity that has received considerable interest in the last two decades. Optimization is the process where designers produce better design saving resources such as time and money. The purpose of optimal design is to obtain best feasible design following a set of pre-selected measures of efficiency. The realization of the scarcity of natural resources drives us towards light weight and low cost structures, thus emphasizing the need for weight and cost optimization of structures. Designers prefer to minimize the volume or the weight of the structure by optimization. Many traditionally mathematical optimization algorithms have been used in structural optimization problems. However, most of these algorithms are limited for the structure design. Recently, evolutionary algorithms (EAs) such as genetic algorithms, evolutionary programming and evolution strategies have attracted designers' community to look for optimal solutions. As these strategies do not apply mathematical assumptions to the optimization problems and have better global search abilities over conventional optimization algorithms. 
The computational drawbacks of mathematical methods (i.e., complex derivatives, sensitivity to initial values, and the large amount of enumeration memory required) have forced researchers to rely on meta-heuristic algorithms based on simulations to solve optimization problems. The common factor in meta-heuristic algorithms is that they combine rules and randomness to imitate natural phenomena. In the last decade, these meta-heuristic algorithms, especially the genetic algorithm have been broadly applied to solve various structural optimization problems, and have occasionally overcome several lacunae of conventional mathematical methods. Genetic algorithms are considered powerful in terms of global optimization, but they have several drawbacks regarding local searches; Tazawa et al. [15] have identified two of them as lack of local search ability and premature convergence. Researchers have experimented with immune based algorithms to overcome these particular drawbacks.

Optimal design of truss structures has always been a fast developing area of research in the field of engineering optimization and has made notable progress in the last decade. Numerous optimization techniques and methodologies have been developed to find optimal truss structures, especially biological-inspired methods imitating natural phenomena and physical processes. Among these are simulated annealing [13], particle swarm optimization [9], Harmony search [11], Genetic algorithms [3,8].

Optimization of truss structures can be classified into three main categories: sizing, configuration, and topology optimization. In line with many researchers, sizing optimization is considered here where cross-sectional area of each member is used as design variables. In addition, the coordinates of the nodes and connectivity among various members are unchanging. Further, most practical design task require that the sizing of variables be chosen from a list of discrete commercial values as opposed to continuous values. This results in a discrete optimization problem of greater complexity. However, this is not an issue for genetic algorithm or immune algorithm methods due to their binary coded nature. Genetic Algorithm and Immune algorithm theories can be equally applied to continuous optimization problems. Optimal design of truss structures involves multi modal search space and design variables capable of taking wide range of values.

Current work presents multi modal optimization technique for the truss optimization based on another such relatively new evolutionary computation paradigm -Artificial Immune System (AIS) [6]. One of the most extensively used AIS model is the CLONal Selection ALGorithm (CLONALG). The computational model of the CLONALG [5] borrows heavily from immunological theory. The CLONALG has been broadly used for solving various real world problems [7]. Coello and Cruz-Corted [4] and Omkar et al. [14] used CLONALG for multi objective optimization and composite design optimization problems respectively. Since most of the real world problems include one or more constraints, suitable constraint handling techniques need to embedded in the algorithm to obtain the solution in the feasible space. The most popular mechanism adopted by researchers is penalty function that allows incorporating constraints into the fitness function. Though they are popular, it is very difficult to determine the value of penalty factors and performance of the algorithm completely depends on them. Aragon et al. [1] developed an algorithm based on new mutation operator which produces large and small step sizes for the search variables. 
The main aim of the present paper is to carry out the systematic comparative study and assess the performance of Artificial Immune System (AIS) approach for 2D/3D truss optimization problems. The work presents the background of the problem, the solution strategies used, their implementation and the results obtained. Finally results are compared with other approaches.

\section{BACKGROUND: ARTIFICIAL IMMUNE SYSTEM (AIS)}

\subsection{Clonal Selection Theory}

Clonal selection theory is developed to explain how an immune response is performed when antigens are recognized by a B cell. The main goal of the immune system is to protect the human body from the attack of foreign organisms. The immune system is capable of distinguishing between the normal components of our organism and the foreign material that can cause us harm. These foreign organisms are called antigens. The molecules called antibodies play the main role on the immune system response. The immune response is specific to a certain foreign organism (antigen). When an antigen is detected, those antibodies that best recognize an antigen will proliferate by cloning. This process is called clonal selection theory. The main features of the clonal selection theory are explored as follows:

- Generation of new random genetic changes subsequently expressed as diverse antibody patterns by a form of accelerated somatic mutation.

- $\quad$ Phenotypic restriction and retention of one pattern to one differentiated cell (clone).

- $\quad$ Proliferation and differentiation on contact of cells with antigens.

\subsection{Clonal Selection Algorithm}

De Castro \& Von Zuben [6] proposed a Clonal selection algorithm named CLONALG, to fulfill these basic processes involved in clonal selection. Initially algorithm proposed to pattern recognition and machine learning tasks but later adapted to perform optimization.

The clonal selection algorithm can be described as follows in a pseudo code:

Begin CLONALG

INPUT: Parameter values.

Generate $j$ antibodies randomly.

Repeat

- Determine affinity of each antibody (objective function value.)

- Sort antibodies using their affinity values from highest to lowest.

- Clone antibodies. Number of clones $\left(N C_{i}\right)=\sum_{i=1}^{j}$ (int) $\left(\beta *^{*} / i\right)$. $\beta$ is multiplication factor. 
- Clones are subjected to hyper mutation process inversely proportional to their affinity.

- Determine the affinity of mutated clones.

- Select j highest affinity clones to compose the new antibodies population

- Replace d lowest affinity antibodies by new individuals generated at random

Until maximum generations

OUTPUT: Best antibody

End CLONALG

\subsection{Constraint handling Technique}

Aragon et al. [1] developed an algorithm based on new mutation operator which produces large and small step sizes for the search variables. The changes that are proposed by the author are

- Process of determining highest affinity antibodies.

- The hyper-mutation operator which depends on the antibodies feasibility: If the antibodies are feasible then the mutation is small. However, for infeasible antibodies, it randomly applies a small or a large mutation.

The algorithm can be described as follows in a pseudo code.

Begin Constrained CLONALG

INPUT: Parameter values.

Generate j antibodies randomly.

Repeat

- Determine affinity of each antibody. The feasible antibodies are in the top of the list followed by infeasible ones. Into the feasible group the best are those with highest objective function values. Into the infeasible antibodies, those with less constraint violation value are the best.

- Sort antibodies using their affinity values from highest to lowest.

- Clone antibodies. Number of clones $\left(N C_{i}\right)=\sum_{i=1}^{j}(\mathrm{int})(\beta * j / i)$. $\beta$ is multiplication factor.

- Clones are subjected to hyper mutation process depending on the clones' feasibility.

- For feasible clones' only single position of the string is changed which is chosen randomly but for the clones which are infeasible clones are mutated with any of the following two equations with $50 \%$ probability. 


$$
\begin{aligned}
& \mathrm{o} \quad x_{i}=x_{i} \pm \operatorname{rand}(0,1) * \frac{\operatorname{range}\left(x_{i}\right)}{\text { generation }} * N C \\
& \mathrm{o} \quad x_{i}=x_{i} \pm \operatorname{rand}(0,1) * \frac{\operatorname{range}\left(x_{i}\right)}{\text { generation } * N C}
\end{aligned}
$$

Where range $\left(x_{i}\right)$ is random number in the range of the variable, generation is the current generation and $N C$ is the number of clones.

- Determine the affinity of mutated clones.

- Select $j$ highest affinity clones to compose the new antibodies population

- Replace d lowest affinity antibodies by new individuals generated at random

\section{Until maximum generations}

OUTPUT: Best antibody

End Constrained CLONALG

\section{NUMERICAL EXAMPLES}

Standard numerical examples that were used in previous truss size optimization papers are considered in this study. The MATLAB computer program was developed to demonstrate the efficiency and robustness of the CLONALG algorithm. These 2D/3D truss structures are analyzed using the FEM displacement method. For all examples presented in this study, the CLONALG algorithm parameters are set as follows: Number of antibodies $=100, \beta=0.5$, replacement factor $=10 \%$ and Maximum generations $=500$.

The objective function is the weight of the structure and constraints considered are stress limitations in the members and displacement limitations of nodes in all directions. The cross-section areas of members are design variables which are continuous over the domain. There is also limitation on minimum cross-section that has to be used. Objective function is

$$
\mathrm{Z}=\sum_{\mathrm{i}=1}^{\mathrm{m}} A_{i} L_{i} \rho_{i}
$$

Where $A_{i}, L_{i}, \rho_{i}$ are cross-section area, length and density of $i^{\text {th }}$ member respectively.

\subsection{0-Bar Planar Truss}

The truss containing 10 members and 6 nodes is shown in the Fig. 1. Two of the nodes are restrained to move totally and the truss is subjected to two loading cases. 


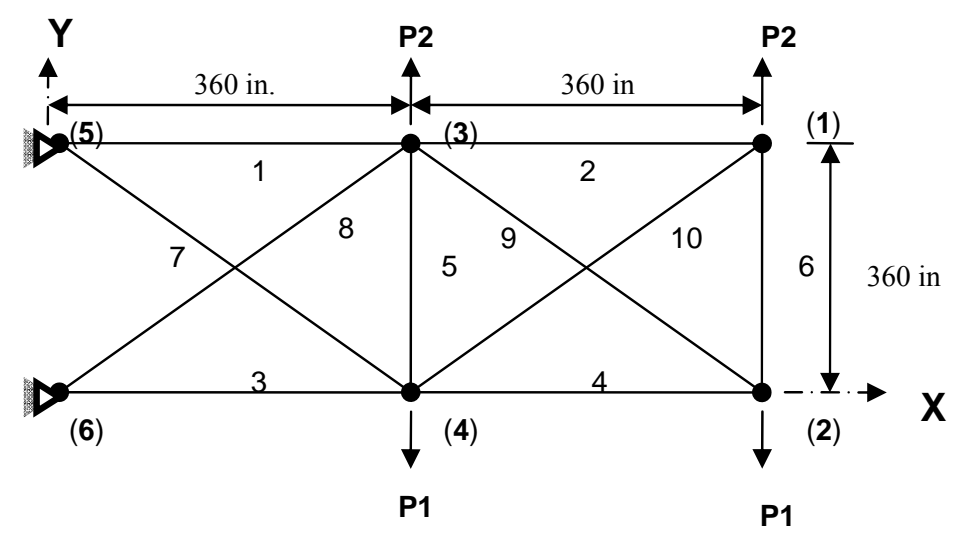

Figure 1: 10-bar planar truss

- $\quad$ Case 1: Single loading condition of $\mathrm{P} 1=100$ kips and $\mathrm{P} 2=0$

- Case 2: Single loading condition of P1 $=150$ kips and P2 $=50$ kips

- The modulus of elasticity and material density of all the members are $10000 \mathrm{ksi}$ and $0.11 \mathrm{~b} /$ in. $^{3}$, respectively.

- $\quad$ Stress limitation of $\pm 25 \mathrm{ksi}$ is imposed on all members

- Displacement limitation of \pm 2 in. is imposed on all the nodes in all directions.

- The design variables are cross-section areas of the members which are continuous over the domain and minimum cross-section area of the member is $0.1 \mathrm{in}^{2}$.

The results obtained in the present study are compared with the outcome of other researchers. The optimal design values obtained using CLONALG for Cases 1 and 2 are given in the last columns of Tables 1 and 2 respectively.

Table 1: Optimal design comparison for 10-bar planar truss (Case 1)

\begin{tabular}{|c|c|c|c|c|c|c|c|c|}
\hline \multicolumn{2}{|l}{ Variables } & \multicolumn{7}{|c|}{ Optimal Cross-section areas (in. ${ }^{2}$ ) } \\
\cline { 3 - 9 } & $\begin{array}{c}\text { GA } \\
{[\mathbf{2}]}\end{array}$ & $\begin{array}{c}\text { HS } \\
{[\mathbf{1 1}]}\end{array}$ & $\begin{array}{c}\text { PSO } \\
{[\mathbf{1 2}]}\end{array}$ & $\begin{array}{c}\text { PSOPC } \\
{[\mathbf{1 2}]}\end{array}$ & $\begin{array}{c}\text { HPSO } \\
{[\mathbf{1 2}]}\end{array}$ & $\begin{array}{c}\text { HPSACO } \\
{[\mathbf{1 0}]}\end{array}$ & $\begin{array}{c}\text { Present Study } \\
\text { CLONALG }\end{array}$ \\
\hline $\mathbf{1}$ & A1 & 28.92 & 30.15 & 33.469 & 30.569 & 30.074 & 30.307 & $\mathbf{3 0 . 3 6}$ \\
\hline $\mathbf{2}$ & A2 & 0.10 & 0.102 & 0.110 & 0.100 & 0.100 & 0.100 & $\mathbf{0 . 1 0}$ \\
\hline 3 & A3 & 24.07 & 22.71 & 23.117 & 22.974 & 23.167 & 23.434 & $\mathbf{2 2 . 9 8}$ \\
\hline $\mathbf{4}$ & A4 & 13.96 & 15.27 & 15.475 & 15.148 & 15.183 & 15.505 & $\mathbf{1 5 . 1 9}$ \\
\hline $\mathbf{5}$ & A5 & 0.10 & 0.102 & 3.649 & 0.100 & 0.100 & 0.100 & $\mathbf{0 . 1 0}$ \\
\hline $\mathbf{6}$ & A6 & 0.56 & 0.544 & 0.116 & 0.547 & 0.551 & 0.5241 & $\mathbf{0 . 5 8}$ \\
\hline $\mathbf{7}$ & A7 & 7.69 & 7.541 & 8.328 & 7.493 & 7.460 & 7.4365 & $\mathbf{7 . 5 2}$ \\
\hline $\mathbf{8}$ & A8 & 21.95 & 21.56 & 23.340 & 21.159 & 20.978 & 21.079 & $\mathbf{2 1 . 4 1}$ \\
\hline $\mathbf{9}$ & A9 & 22.09 & 21.45 & 23.014 & 21.556 & 21.508 & 21.229 & $\mathbf{2 1 . 3 9}$ \\
\hline $\mathbf{1 0}$ & A10 & 0.10 & 0.100 & 0.190 & 0.100 & 0.100 & 0.100 & $\mathbf{0 . 1 0 0}$ \\
\hline \multicolumn{2}{|l}{ Weight (lb) } & $\mathbf{5 0 7 6 . 3 1}$ & $\mathbf{5 0 5 7 . 8 8}$ & $\mathbf{5 5 2 9 . 5 0}$ & $\mathbf{5 0 6 1 . 0 0}$ & $\mathbf{5 0 6 0 . 9 2}$ & $\mathbf{5 0 5 6 . 5 6}$ & $\mathbf{5 0 5 9 . 4 2}$ \\
\hline
\end{tabular}


Table 2: Optimal design comparison for 10-bar planar truss (Case 2)

\begin{tabular}{|c|c|c|c|c|c|c|c|}
\hline \multicolumn{2}{|l|}{ Variables } & \multicolumn{6}{|c|}{ Optimal Cross-section areas (in. ${ }^{2}$ ) } \\
\cline { 2 - 8 } & $\begin{array}{c}\text { HS } \\
{[\mathbf{2}]}\end{array}$ & $\begin{array}{c}\text { PSO } \\
{[\mathbf{1 1}]}\end{array}$ & $\begin{array}{c}\text { PSOPC } \\
{[\mathbf{1 1}]}\end{array}$ & $\begin{array}{c}\text { HPSO } \\
{[\mathbf{1 1}]}\end{array}$ & $\begin{array}{c}\text { HPSACO } \\
{[\mathbf{1 0}]}\end{array}$ & $\begin{array}{c}\text { Present Study } \\
\text { CLONALG }\end{array}$ \\
\hline $\mathbf{1}$ & A1 & 23.25 & 22.935 & 23.473 & 23.353 & 23.194 & $\mathbf{2 2 . 7 2}$ \\
\hline $\mathbf{2}$ & A2 & 0.102 & 0.113 & 0.101 & 0.100 & 0.100 & $\mathbf{0 . 1 0 0}$ \\
\hline 3 & A3 & 25.73 & 25.355 & 25.287 & 25.502 & 24.585 & $\mathbf{2 4 . 9 1}$ \\
\hline $\mathbf{4}$ & A4 & 14.51 & 14.373 & 14.413 & 14.250 & 14.221 & $\mathbf{1 4 . 4 0}$ \\
\hline 5 & A5 & 0.100 & 0.100 & 0.100 & 0.100 & 0.100 & $\mathbf{0 . 1 0}$ \\
\hline $\mathbf{6}$ & A6 & 1.977 & 1.990 & 1.969 & 1.972 & 1.969 & $\mathbf{1 . 9 7}$ \\
\hline $\mathbf{7}$ & A7 & 12.21 & 12.346 & 12.362 & 12.363 & 12.489 & $\mathbf{1 2 . 5 2}$ \\
\hline $\mathbf{8}$ & A8 & 12.61 & 12.923 & 12.694 & 12.894 & 12.925 & $\mathbf{1 3 . 6 0 1}$ \\
\hline $\mathbf{9}$ & A9 & 20.36 & 20.678 & 20.323 & 30.356 & 20.952 & $\mathbf{2 0 . 2 5 3}$ \\
\hline $\mathbf{1 0}$ & A10 & 0.100 & 0.100 & 0.103 & 0.101 & 0.101 & $\mathbf{0 . 1 0 0}$ \\
\hline Weight (lb) & $\mathbf{4 6 6 8 . 8 1}$ & $\mathbf{4 6 7 9 . 4 7}$ & $\mathbf{4 6 7 7 . 7 0}$ & $\mathbf{4 6 7 7 . 2 9}$ & $\mathbf{4 6 7 5 . 7 8}$ & $\mathbf{4 6 7 7 . 1 2}$ \\
\hline
\end{tabular}

The minimum weight is 5059.42 and 4677.12 in Cases 1 and 2 respectively. Even though the weight is a little higher compare to other researchers, it is observed that results of other researchers violated constraints slightly.

\subsection{5-Bar Space Truss}

The truss containing 25 members and 10 nodes is shown in Figure 2. Four of the nodes are restrained to move totally and the truss is subjected to two loading conditions acting on the 1st, 2nd, 3rd and 6th node of the truss (Table 3).

- The modulus of elasticity and material density of all the members are $10000 \mathrm{ksi}$ and $0.11 \mathrm{~b} /$ in. $^{3}$, respectively.

- The 25 members are linked into eight groups (Table 4), two groups with a single member and another with seven members.

- Stress limitation imposed on the mebers are given in the Table 4 and displacement limitation of $\pm 0.35 \mathrm{in}$. is imposed on all the nodes in all directions.

- The design variables are cross-section areas of the members which are continuous over the domain and minimum cross-section area of the member is $0.01 \mathrm{in}^{2}$.

The optimal design values obtained using CLONALG are tabulated in last column of Table 5 for comparison with the outcome of other researchers. 


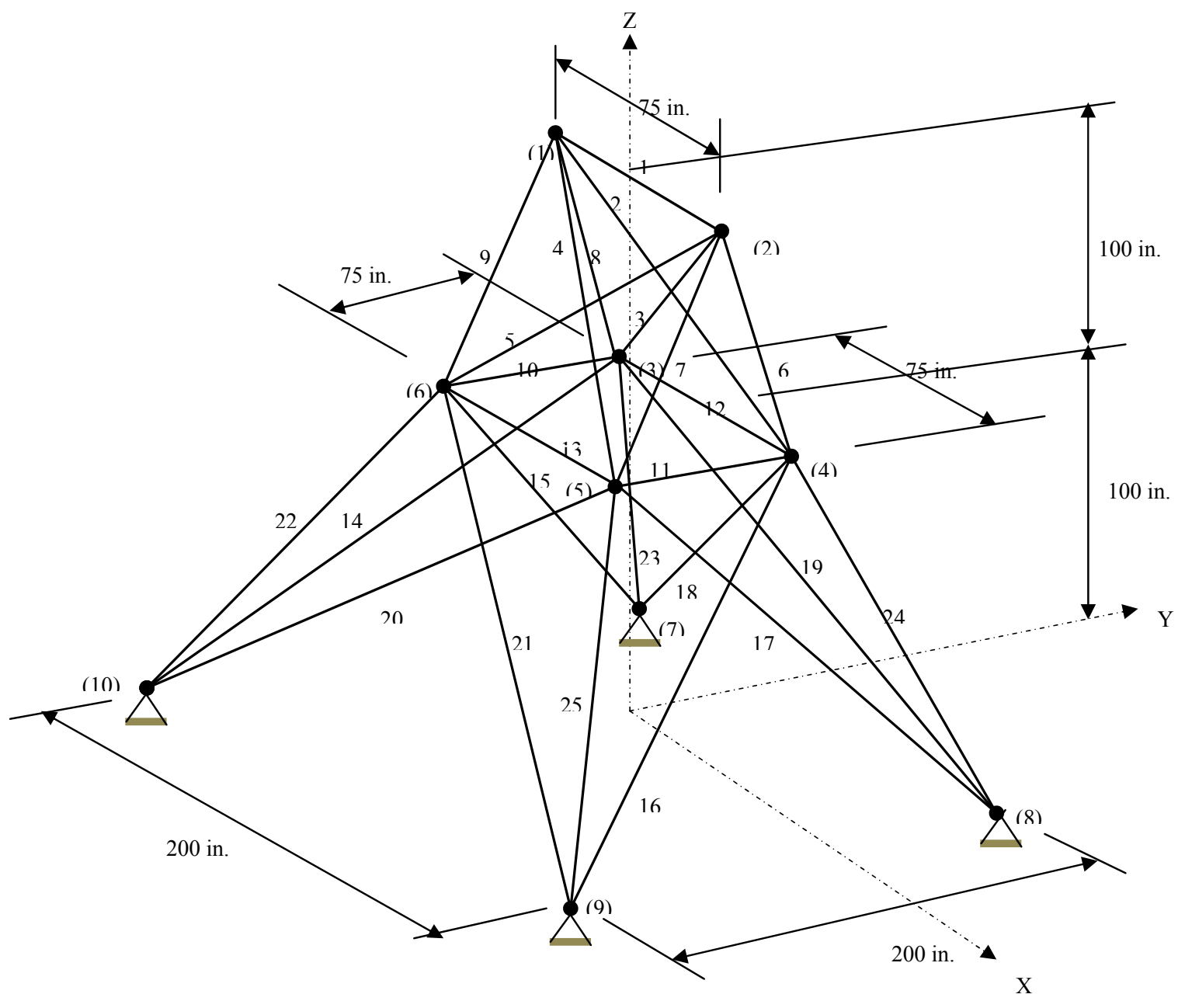

Figure 2: 25-bar space truss

Table 3: Loading conditions for 25-bar space truss

\begin{tabular}{|l|l|l|l|l|l|l|}
\hline \multirow{2}{*}{ Node } & \multicolumn{3}{|l|}{ Condition 1 } & \multicolumn{3}{c|}{ Condition 2 } \\
\cline { 2 - 7 } & $\mathrm{P}_{\mathrm{x}}$ & $\mathrm{P}_{\mathrm{y}}$ & $\mathrm{P}_{\mathrm{z}}$ & $\mathrm{P}_{\mathrm{x}}$ & $\mathrm{P}_{\mathrm{y}}$ & $\mathrm{P}_{\mathrm{z}}$ \\
\hline $\mathbf{1}$ & 0 & -20 & -5 & 1 & 10 & -5 \\
\hline $\mathbf{2}$ & 0 & -20 & -5 & 0 & 10 & -5 \\
\hline $\mathbf{3}$ & 0 & 0 & 0 & 0.5 & 0 & 0 \\
\hline $\mathbf{6}$ & 0 & 0 & 0 & 0.5 & 0 & 0 \\
\hline
\end{tabular}


Table 4: Member stress limitations for the 25-bar space truss.

\begin{tabular}{|c|c|c|c|}
\hline \multicolumn{2}{|c|}{ Variables } & $\begin{array}{c}\text { Compressive Stress } \\
\text { Limitations (ksi) }\end{array}$ & $\begin{array}{c}\text { Tensile Stress } \\
\text { Limitations (ksi) }\end{array}$ \\
\hline 1 & A1 & 35.092 & 40 \\
\hline $\mathbf{2}$ & A2 A5 & 11.590 & 40 \\
\hline 3 & A6 A9 & 17.305 & 40 \\
\hline $\mathbf{4}$ & A10 A11 & 35.092 & 40 \\
\hline $\mathbf{5}$ & A12 A13 & 35.092 & 40 \\
\hline 6 & A14 A17 & 6.759 & 40 \\
\hline 7 & A18 A21 & 6.959 & 40 \\
\hline 8 & A22 A25 & 11.082 & 40 \\
\hline
\end{tabular}

Table 5: Optimal design comparison for 25-bar space truss

\begin{tabular}{|c|c|c|c|c|c|c|c|c|}
\hline \multirow{2}{*}{} & Variables & \multicolumn{7}{|c|}{ Optimal Cross-section areas (in. ${ }^{2}$ ) } \\
\cline { 3 - 9 } & $\begin{array}{c}\text { ACO } \\
{[\mathbf{2}]}\end{array}$ & $\begin{array}{c}\text { HS } \\
{[\mathbf{1 1}]}\end{array}$ & $\begin{array}{c}\text { PSO } \\
{[\mathbf{1 2}]}\end{array}$ & $\begin{array}{c}\text { PSOPC } \\
{[\mathbf{1 2}]}\end{array}$ & $\begin{array}{c}\text { HPSO } \\
{[\mathbf{1 2}]}\end{array}$ & $\begin{array}{c}\text { HPSACO } \\
{[\mathbf{1 0}]}\end{array}$ & $\begin{array}{c}\text { Present } \\
\text { Study } \\
\text { CLONALG }\end{array}$ \\
\hline $\mathbf{1}$ & $\mathrm{A} 1$ & 0.010 & 0.047 & 9.863 & 0.010 & 0.010 & 0.010 & $\mathbf{0 . 0 3}$ \\
\hline $\mathbf{2}$ & $\mathrm{A} 2 \sim \mathrm{A} 5$ & 2.000 & 2.022 & 1.798 & 1.979 & 1.970 & 2.054 & $\mathbf{1 . 9 2}$ \\
\hline $\mathbf{3}$ & $\mathrm{A} 6 \sim \mathrm{A} 9$ & 2.966 & 2.950 & 3.654 & 3.011 & 3.016 & 3.088 & $\mathbf{3 . 1 2}$ \\
\hline $\mathbf{4}$ & $\mathrm{A} 10 \sim \mathrm{A} 11$ & 0.010 & 0.010 & 0.100 & 0.100 & 0.010 & 0.010 & $\mathbf{0 . 0 3}$ \\
\hline $\mathbf{5}$ & $\mathrm{A} 12 \sim \mathrm{A} 13$ & 0.012 & 0.014 & 0.100 & 0.100 & 0.010 & 0.010 & $\mathbf{0 . 0 1}$ \\
\hline $\mathbf{6}$ & $\mathrm{A} 14 \sim \mathrm{A} 17$ & 0.689 & 0.688 & 0.596 & 0.657 & 0.694 & 0.679 & $\mathbf{0 . 6 5}$ \\
\hline $\mathbf{7}$ & $\mathrm{A} 18 \sim \mathrm{A} 21$ & 1.679 & 1.657 & 1.659 & 1.678 & 1.681 & 1.611 & $\mathbf{1 . 6 8}$ \\
\hline $\mathbf{8}$ & A22 A25 & 2.668 & 2.663 & 2.612 & 2.693 & 2.643 & 2.678 & $\mathbf{2 . 6 7 4}$ \\
\hline Weight (lb) & $\mathbf{5 4 5 . 5 3}$ & $\mathbf{5 4 4 . 3 8}$ & $\mathbf{6 2 7 . 0 8}$ & $\mathbf{5 4 5 . 2 7}$ & $\mathbf{5 4 5 . 1 9}$ & $\mathbf{5 4 4 . 9 9}$ & $\mathbf{5 4 5 . 8 2}$ \\
\hline
\end{tabular}

The solution converged in less than 75 iterations and the best solution is obtained in 73rd iteration. The weight is very little higher compare to other works but this method found solutions in feasible zone. It is observed that results of other researchers violate the constraints lightly.

\section{CLOSING REMARKS}

In this work a constrained multi modal optimization algorithm (Constrained CLONALG) based on the principles of immune systems is developed to determine the optimal weight of 2D/3D truss structures. Exploration and Exploitation of feasible solutions in constrained space are realized by employing modified mutation operator in the algorithm. The results are compared with the other conventional mathematical approaches and evolutionary techniques. Even though present solutions in few cases are a little higher compare to other works but it handled constrained very strictly. It was observed that some of results of other researchers were in the infeasible zone. It was important to note that very little 
work is done so far regarding the use of AIS in constrained optimization problems and further work needs to be done to improve the quality of solutions. The method proposed in this study is not limited to truss structural optimization problems that treat continuous sizing variables. It can be easily used for problems with continuous and/or discrete sizing variables. Besides trusses, the CLONALG can be applied to other types of structural optimization problems, including frame structures, plates, and shells.

\section{References}

[1] Aragon, V.S., Esquivel, S.C., Coello, C.A.: Artificial Immune System for Solving Constrained Optimization Problem. Revista Iberoamericana de Inteligencia Artificial. 35, 55-66 (2007)

[2] Camp, C., Bichon, J.: Design of space trusses using ant colony optimization. J. Struct Engg ASCE. 130(5), 741-51 (2004)

[3] Coello, CA, Christiansen AD.: Multi-objective optimization of trusses using genetic algorithm .J. Comput Struct. Vol. 75(6), pp.647-60 (2000)

[4] Coello, N., Cruz-Cortes, N.: Multi objective optimizations using ideas from the clonal selection principle. In: Genetic and Evolutionary Computational Conference, GECCO 2003, Lecture notes in computer science. Vol 2723, Springer, Berlin, pp 158-170 (2003)

[5] De Castro, L.N., Von Zuben, F. J.: Learning and optimization using the clonal selection principle. IEEE transactions on evolutionary computation. 63, 239-251 (2002)

[6] De Castro, L.N., Von Zuben, F. J.: Artificial immune systems: Part 1. Basic theory and applications. Technical report RT DCA. 01/99 (1999)

[7] De Castro, L.N., Von Zuben, F. J.: The Clonal Selection Algorithm with Engineering Applications. In: GECCO'00, Workshop on Artificial Immune Systems and Their Applications. 36-37 (2000)

[8] Deb K, Gulati, S.: Design of truss-structures for minimum weight using genetic algorithms. J. Finite Elem Anal Design. 37,447-65 (2001)

[9] Fourie, P.C, Groenwold, A.A.: The particle swarm optimization algorithm in size and shape optimization. J. Struct Multidisc Optim.23,259-67 (2002)

[10] Kaveh, A., Talatahari, S.: Particle swarm optimizer, ant colony strategy and harmony search scheme hybridized for optimization of truss structures. J. Comput Struct. $87,267-283$ (2009)

[11] Lee, K.S., Geem, Z.W.: A New Structural Optimization Method Based on the Harmony Search Algorithm. J. Comput. Struct. Vol. 82(9/10), pp.781-798 (2004)

[12] Li, L.J., Huand, Z.B., Liu, F., Wu, Q.H.: A heuristic particle swarm optimizer for optimization of pin connected structures . J.comput struct . 85, 340-9 (2007) 
[13] Moh, J.S., Chiang, D.Y.: Improved simulated annealing search for structural optimization. J. AIAA. 38(10), 1965-73 (2000)

[14] Omkar, S.N., Khandelwal, R., Yathindra, S., Naik, N., Gopalakrishnan, G.S.: Artificial immune system for multi-objective design optimization of composite structures $\mathrm{J}$. Engineering Applications of Artificial Intelligence. 21, 1416-1429 (2008)

[15] Tazawa, I., Koakutsu. S., Hirata. H.: An immunity based genetic algorithm and its application to the VLSI floorplan design problem. Proceedings IEEE International conference on evolution computing, 417-21 (1996)

\section{Abbreviations}

ACO: Any Colony Optimizer

GA: Genetic Algorithms

HS: Harmony Search

PSO: Particle Swarm Optimizer

PSOPC: Particle Swarm Optimizer with Passive Congregation

HPSO: Heuristic Particle Swarm Optimizer 